\title{
Intraethnic variation in steroid-5-alpha-reductase polymorphisms in prostate cancer patients: a potential factor implicated in 5-alpha-reductase inhibitor treatment
}

\author{
LUIS ALBERTO HENRÍQUEZ-HERNÁNDEZ ${ }^{1,2,3 *}$, ALMUDENA VALENCIANO², PALMIRA FORO-ARNALOT ${ }^{4}$, \\ MARÍA JESÚS ÁLVAREZ-CUBERO ${ }^{5,6}$, JOSÉ MANUEL COZAR ${ }^{7}$, JOSÉ FRANCISCO SUÁREZ-NOVO ${ }^{8}$, \\ MANEL CASTELLS-ESTEVE ${ }^{8}$, PABLO FERNÁNDEZ-GONZALO $^{9}$, BELÉN DE-PAULA-CARRANZA $^{9}$, \\ MONTSE FERRER ${ }^{10}$, FERRÁN GUEDEA ${ }^{11}$, GEMMA SANCHO-PARDO $^{12}$, JORDI CRAVEN-BARTLE ${ }^{12}$, \\ MARÍA JOSÉ ORTIZ-GORDILLO ${ }^{13}$, PATRICIA CABRERA-ROLDÁN ${ }^{13}$, ESTEFANÍA HERRERA-RAMOS ${ }^{14,15}$, \\ CARLOS RODRÍGUEZ-GALLEGO ${ }^{14,15}$ and PEDRO C. LARA ${ }^{1,2,3}$ \\ ${ }^{1}$ Radiation Oncology Department, Hospital Universitario de Gran Canaria Dr. Negrín, Las Palmas 35010, Spain \\ ${ }^{2}$ Instituto Canario de Investigación del Cáncer, Las Palmas 38204, Spain \\ ${ }^{3}$ Clinical Sciences Department, Universidad de Las Palmas de Gran Canaria, Las Palmas 35016, Spain \\ ${ }^{4}$ Institud d'Oncologia Radioteràpica, Hospital de la Esperanza, Parc de Salut Mar, Barcelona 08003, Spain \\ ${ }^{5}$ Laboratory of Genetic Identification, Legal Medicine and Toxicology Department, Facultad de Medicina, \\ Universidad de Granada, Granada 18012, Spain \\ ${ }^{6}$ GENYO, Pfizer-University of Granada-Andalusian Government Centre for Genomics and Oncological Research, \\ Granada 18016, Spain \\ ${ }^{7}$ Department of Urology, Hospital Universitario Virgen de las Nieves, Granada 18014, Spain \\ ${ }^{8}$ Department of Urology, Hospital Universitari de Bellvitge, L'Hospitalet de Llobregat 08907, Spain \\ ${ }^{9}$ Radiation Oncology Department, Onkologikoa, Guipuzcoa 20014, Spain \\ ${ }^{10}$ Health Services Research Group, Institut de Recerca Hospital del Mar (IMIM), Barcelona 08003, Spain \\ ${ }^{11}$ Department of Radiation Oncology, Institut Català d'Oncologia (ICO), L'Hospitalet de Llobregat 08907, Spain \\ ${ }^{12}$ Radiation Oncology Department, Hospital de la Santa Creu i Sant Pau, Barcelona 08026, Spain \\ ${ }^{13}$ Radiation Oncology Department, Hospital Universitario Virgen del Rocío, Sevilla 41013, Spain \\ ${ }^{14}$ Department of Immunology, Hospital Universitario de Gran Canaria Dr. Negrín, Las Palmas 35010, Spain \\ ${ }^{15}$ Department of Medical and Surgical Sciences, Universidad de Las Palmas de Gran Canaria, Las Palmas 35016, Spain
}

[Henríquez-Hernández L. A., Valenciano A., Foro-Arnalot P., Álvarez-Cubero M. J., Cozar J. M., Suárez-Novo J. F., Castells-Esteve M.,
Fernández-Gonzalo P., De-Paula-Carranza B., Ferrer M., Guedea F., Sancho-Pardo G., Craven-Bartle J., Ortiz-Gordillo M. J., Cabrera-Roldán P.,
Herrera-Ramos E., Rodríguez-Gallego C. and Lara P. C. 2015 Intraethnic variation in steroid-5-alpha-reductase polymorphisms in prostate
cancer patients: a potential factor implicated in 5-alpha-reductase inhibitor treatment. J. Genet. 94, 335-341]

\section{Introduction}

Steroid-5-alpha-reductase inhibitors are commonly used in the treatment of benign prostatic enlargement and male alopecia with controversial results. Ethnic diversity is an important factor, accounting for interindividual variation in drug responsiveness. The aim of this study was to evaluate the genotypic distribution of 22 SNPs in steroid-5-alphareductase alpha polypeptides 1 and 2 in a set of 601 prostate cancer patients from four different Spanish regions. The genotyping was done in a Biotrove OpenArray NT Cycler.

\footnotetext{
*For correspondence. E-mail: lhenriquez@dcc.ulpgc.es.
}

Twelve SNPs of 22 analysed were differentially distributed among the different regions. Since all subjects were Caucasian of Spanish origin, this result showed differences in genotype distribution among subject from the same ethnic origin. These observations were confirmed in cluster analysis, principal component analysis and in the differential distribution of haplotypes among the populations. Differences in distribution of genotypes within different populations of the same ethnicity could be an important factor responsible for the wide variety of pharmacological responses to steroid-5-alpha-reductaseinhibitors.

Prostate cancer $(\mathrm{PCa})$ is a hormone-dependent tumour which needs androgens for disease initiation and progression

Keywords. 5-alpha-reductase polymorphism; 5-alpha-reductase inhibitors; OpenArray; prostate cancer; pharmacoethnicity. 
(Huggins 1967). Although, testosterone (T) is the most abundant serum androgen, dihydrotestosterone (DHT) is the main prostate androgen. $\mathrm{T}$ is converted to DHT by the enzyme steroid-5-alpha-reductase (SRD5A) in the prostate, testes, hair follicles and adrenal glands (McConnell 1995). Since the discovery of the implications of SRD5A2 deficiency, it has been an increasing interest in the development of steroid5 -alpha-reductase inhibitors ( $5 \alpha$-RI). Among several compounds, only two drugs have been FDA approved for clinical use: finasteride, which inhibits SRD5A2 and was approved for the treatment of benign prostatic enlargement (BPE) and male alopecia; and dutasteride, which inhibits SRD5A1 and SRD5A2 (approved for the treatment of BPE) (Edwards and Moore 2002; Andriole and Kirby 2003). Nowadays, dutasteride and finasteride do not prevent $\mathrm{CaP}$, but merely temporarily shrink tumours that have a low potential for being lethal (Walsh 2010).

The enzymatic activity of SRD5A is highly modulated by single-nucleotide polymorphisms (SNPs) located in these genes (Giwercman et al. 2005; Cussenot et al. 2007). These genetic variations in key genes belonging to the androgen pathway seem to be important for the prostate response to androgens (Lindstrom et al. 2006). We have recently reported that differences in the distribution of genotypes within different populations of the same ethnicity could be an important confounding factor in association studies (Henriquez-Hernandez et al. 2013a).

We designed a study aimed to evaluate the genotypic distribution of 22 SNPs in SRD5A1 and SRD5A2 in a set of Spanish prostate cancer patients, to determine the homogeneity of the population and to disclose potential bias associated with the failure of $5 \alpha$-RI when prescribed.

\section{Materials and methods}

A total of 601 patients with nonmetastatic localized prostate cancer were included in this study. Geographical distribution of patients was as follows (Henriquez-Hernandez et al. 2013a): 91 (15.14\%) from Andalusia, 51 (8.48\%) from Basque Country, $238(39.60 \%)$ from Canary and $221(36.77 \%)$ from Catalonia. All patients were of Spanish origin and all of them received written informed consent before sample collection. Ethnicity of patients was established until the second parental generation. This study was approved by the Research and Ethics Committee of each institution participant in the study.

DNA was isolated from $300 \mu \mathrm{L}$ of whole-blood in an iPrep $^{\mathrm{TM}}$ purification instrument using the iPrep $^{\mathrm{TM}}$ PureLink $^{\mathrm{TM}}$ gDNA Blood kit (Invitrogen, Life Technologies, Carlsbad, USA). DNA integrity was determined by NanoDrop ND-1000 (NanoDrop Technologies, Wilmington, USA).

SNPs were selected using data of individuals with European ancestry (CEU) from the HapMap Project (available at: http://www.hapmap.org). Pairwise linkage disequilibrium (LD) tagging was achieved with Haploview ver. 4.2 software (free downloaded from http://www.broadinstitute.org/ scientific-community/science/programs/medical-and-populationgenetics/haploview/haploview) (Barrett et al. 2005). The $R^{2}$ given by Haploview was $>0.95$ for all genes. Description of SNPs is provided in table 1 in electronic supplementary material at http://www.ias.ac.in/jgenet/.

The SNP genotyping was performed in a Biotrove OpenArray ${ }^{\circledR}$ NT Cycler (Applied Biosystems, Foster City, USA) following the instructions of manufacturer (HenriquezHernandez et al. 2013b). The fluorescence results were read using the OpenArray ${ }^{\circledR}$ SNP genotyping analysis software ver. 1.0.5 (Applied Biosystems). The genotyping analysis was performed using TaqMan Genotyper software ver. 1.0.1 (available at: http://www.invitrogen.com/site/us/en/home/Glo bal/forms/taqman-genotyper-software-download-reg.html) using autocalling as the call method. The quality value of the data points was determined by a threshold above 0.95 . Genotyping analysis was done for each population separately and it was done automatically without any manual assignment of genotypes as previously reported (Henriquez-Hernandez et al. 2013a). All the genotyped samples met the quality criteria and all samples were genotyped with the same batch of material and at the same time.

Genotype and allelic frequencies were determined using the web-based environment SNPator (SNP Analysis To Results, from the Spain's National Genotyping Center and the National Institute for Bioinformatics) (Morcillo-Suarez et al. 2008).

Principal component analysis (PCA) was done using SnpMatrix and XSnpMatrix classes and methods (Clayton 2012), implemented as an $\mathrm{R}$ package and available from Bioconductor (as of ver. 2.11; http://bioconductor.org). Nonsupervised hierarchical clustering of SNP in each population was done using MultiExperiment Viewer ver. 4.9 (available at: http://www.tigr.org).

\section{Results}

A total of 601 PCa patients were genotyped for 22 SNPs; 10 SNPs located in SRD5A1 gene and 12 SNPs located in SRD5A2 gene. Of the 13,222 possible determinations, $94.31 \%$ were successfully genotyped.

The genotypic and allelic frequencies are shown in table 1. The genotype distribution was different among the study populations in 12 of the 22 SNPs: rs166050, rs501999, rs518673, rs3822430, rs8192120, rs39848 (SRD5A1); and rs2208532, rs12470143, rs2281546, rs3754838, rs523349, rs9332975 (SRD5A2) ( $\chi^{2}$ test, table 1), showing a differential distribution of genotypes among populations.

A nonsupervised hierarchical cluster was performed to visualize the genotype distributions among the four populations. For SRD5A1, polymorphisms were distributed into three main clusters, each one with different numbers and identities of SNPs, suggesting heterogeneity among populations (figure 1 in electronic supplementary material, left panel). For SRD5A2, SNPs were distributed into two main clusters, very similar for Basque, Canary and Catalan populations, 
Table 1. Genotype and allelic frequencies of $S R D 5 A 1$ and $S R D 5 A 2$ gene polymorphisms among the populations.

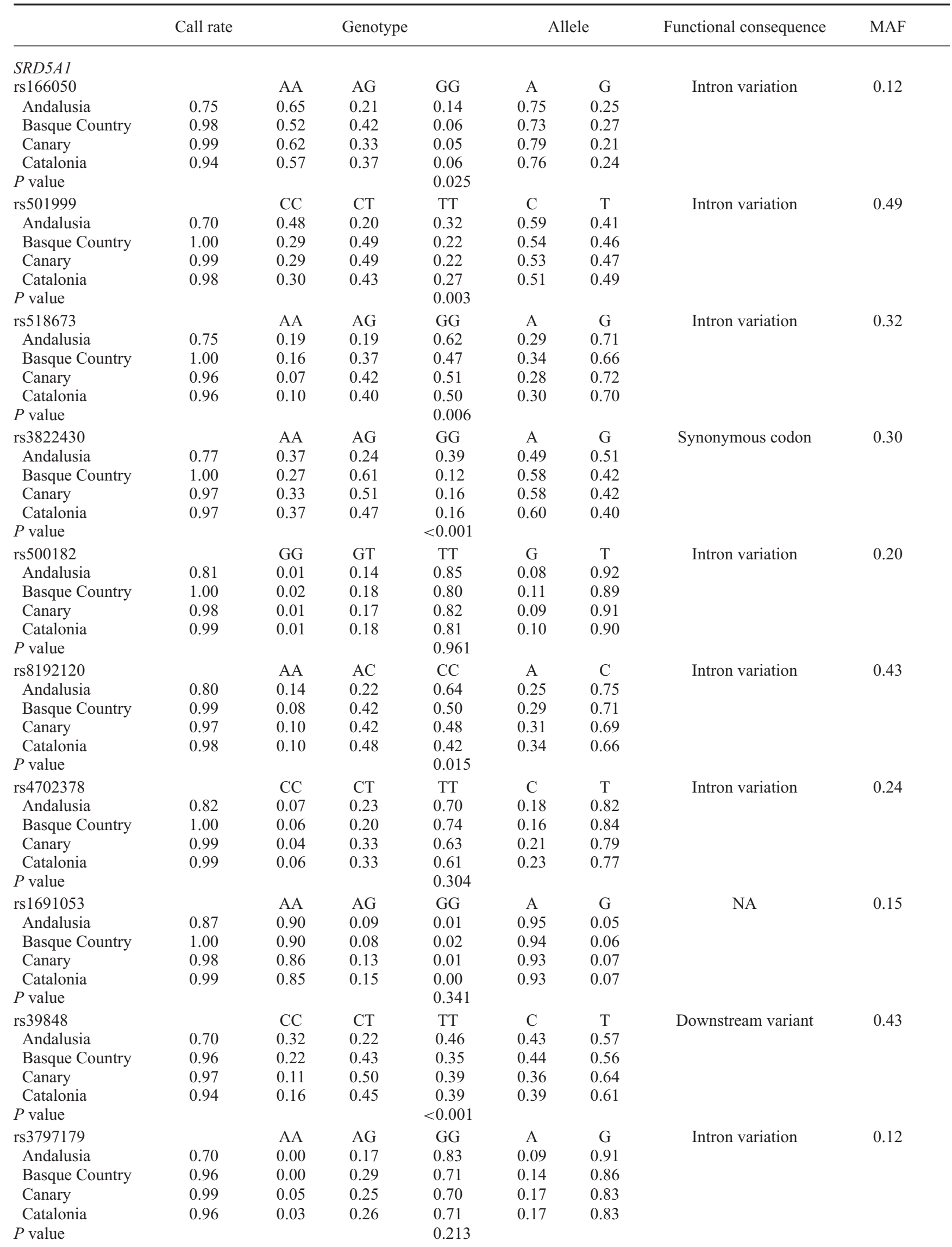


Table 1 (contd)

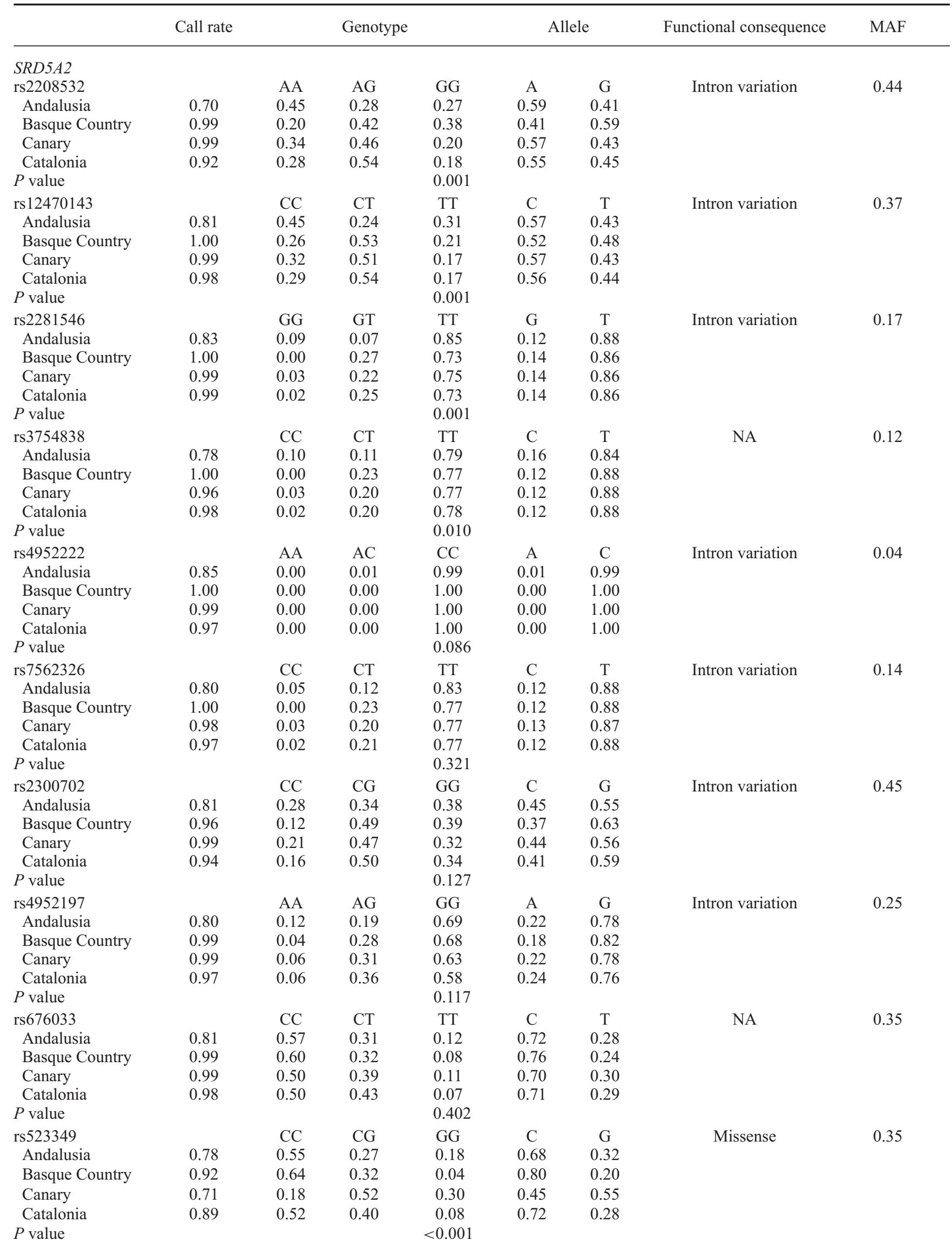


Table 1 (contd)

\begin{tabular}{|c|c|c|c|c|c|c|c|c|}
\hline \multirow[b]{2}{*}{ rs9332975 } & \multirow[t]{2}{*}{ Call rate } & \multicolumn{3}{|c|}{ Genotype } & \multicolumn{2}{|c|}{ Allele } & \multirow{2}{*}{$\begin{array}{c}\text { Functional consequence } \\
\text { utr variant }\end{array}$} & \multirow{2}{*}{$\begin{array}{l}\text { MAF } \\
0.14\end{array}$} \\
\hline & & $\mathrm{CC}$ & $\mathrm{CT}$ & $\mathrm{TT}$ & $\mathrm{C}$ & $\mathrm{T}$ & & \\
\hline Andalusia & 0.77 & 0.10 & 0.07 & 0.83 & 0.14 & 0.86 & & \\
\hline Basque Country & 1.00 & 0.00 & 0.26 & 0.74 & 0.13 & 0.87 & & \\
\hline Canary & 0.99 & 0.03 & 0.19 & 0.78 & 0.13 & 0.87 & & \\
\hline Catalonia & 0.98 & 0.02 & 0.20 & 0.78 & 0.12 & 0.88 & & \\
\hline$P$ value & & & & 0.002 & & & & \\
\hline rs7594951 & & $\mathrm{CC}$ & $\mathrm{CT}$ & $\mathrm{TT}$ & $\mathrm{C}$ & $\mathrm{T}$ & Intron variation & 0.11 \\
\hline Andalusia & 0.75 & 0.84 & 0.10 & 0.06 & 0.89 & 0.11 & & \\
\hline Basque Country & 1.00 & 0.76 & 0.24 & 0.00 & 0.88 & 0.12 & & \\
\hline Canary & 0.99 & 0.77 & 0.20 & 0.03 & 0.88 & 0.12 & & \\
\hline Catalonia & 0.98 & 0.78 & 0.20 & 0.02 & 0.88 & 0.12 & & \\
\hline$P$ value & & & & 0.193 & & & & \\
\hline
\end{tabular}

MAF, minor allele frequency; NA, not available. Functional consequence and MAF are available at: http://www.ncbi.nlm.nih.gov/projects/ $\mathrm{SNP} /$. Differences in the genotype distribution were assessed by $\chi^{2}$ test.

but clearly different from Andalusian population (figure 1 in electronic supplementary material, right panel). PCA was performed to identify global differences among populations. Components 1, 2 and 3 were responsible for the $43.7 \%$, $22.6 \%$ and $15.3 \%$ of the variance for $S R D 5 A 1$, respectively (cumulative percentage: $81.6 \%$ ); and $48.8 \%, 33.2 \%$ and $9.1 \%$ of the variance for $S R D 5 A 2$ gene, respectively (cumulative percentage: $91.1 \%$ ). The first component distinguished between the populations for both genes clearly showed the differences in the distribution of genotypes between the analysed populations (figure 1).

Haplotype analysis was performed in SNPator. For SRD5A1 (chromosome 5), the haplotype GCCTATGGCA was only present among the Andalusian subjects. For SRD5A2 (chromosome 2), there is a greater homogeneity in the distribution of haplotypes. The fact that the most frequent haplotypes were equal in all populations suggests a similarity between individuals of the same ethnicity.

\section{Discussion}

We have recently reported differences in the distribution of genotypes within different populations of the same ethnicity (Henriquez-Hernandez et al. 2013a), highlighting the importance of ethnic-specific differences in drug responsiveness (Yasuda et al. 2008; $\mathrm{Ma}$ and $\mathrm{Lu}$ 2011). In the present paper, we observed that genotype distribution of 12 of 22 SNPs were statistically different among the studied populations. The Spanish population is considered as Caucasian. However, the natural history of each subpopulation is different, and there is a high complexity in the Mediterranean migration processes that impact in the different population sources on the genetic composition of the Spanish population (Ambrosio et al. 2010). While Andalusian population seems to be originated by migrations from Arabian Peninsula, Fertile Crescent, Balkan region and North Africa (Ambrosio et al. 2010), Canary population has been influenced from Northwest Africa migration and European colonization (Rando et al. 1999). Differences among populations were also evident in haplotype analysis, suggesting that each gene need to be considered individually to find possible confounding variables that would be crucial for the interpretation of results. Neither ethnicity nor genetic endowment of patients is taken into account in clinical trials.

$5 \alpha-\mathrm{RI}$ arisen as a promise treatment for PCa prevention, as specific blocker of the enzyme responsible for the synthesis of DHT. We suggest here that SNPs located in SRD5AI and SRD5A2 may be relevant factors to be considered when assessing the response to these drugs. Although, both genes encode for key enzymes in the conversion of testosterone into DHT and represent attractive targets for preventing prostate cancer development, they are expressed differentially in the prostate tissue. Thus, under normal physiologic conditions, SRD5A2 is preferentially expressed over SRD5A1 in the prostate (Thomas et al. 2005; Titus et al. 2005). In prostate cancer cells, however, the balance in expression of these genes shift toward predominant expression of SRD5A1 (Titus et al. 2005; Stanbrough et al. 2006), supporting a role for both enzymes in DHT bioavailability and carcinogenesis. However, the importance of androgens in early cancer initiation is emphasized by the fact that finasteride, a 5-AR type 2 inhibitor, and dutasteride, a dual 5-AR inhibitor targeting both 5-AR type 1 and type 2 enzymes, have been shown that the risk of prostate cancer incidence reduce by almost 23\% (Thompson et al. 2003; Andriole et al. 2010). Since SRD5A1 and SRD5A2 are differentially expressed and are blockaded by different drugs, the role of SNPs in each gene should be studied intensely taking these biological peculiarities in to account. However, the fact that rs523349-a SNP clearly involved in the success of chemoprevention of $\mathrm{CaP}$ using 5-alpha-reductase inhibitors (Cussenot et al. 2007) appeared differentially distributed in our study, reinforce our findings.

The present study has some limitations that should be noted. First, to blind the analysis, no clinical data of patients were available, i.e., there are no data about TNM staging, tumour grade, biochemical failure, or Gleason Score. Second, the study was focussed on SRD5A1 and SRD5A2, 
(a)

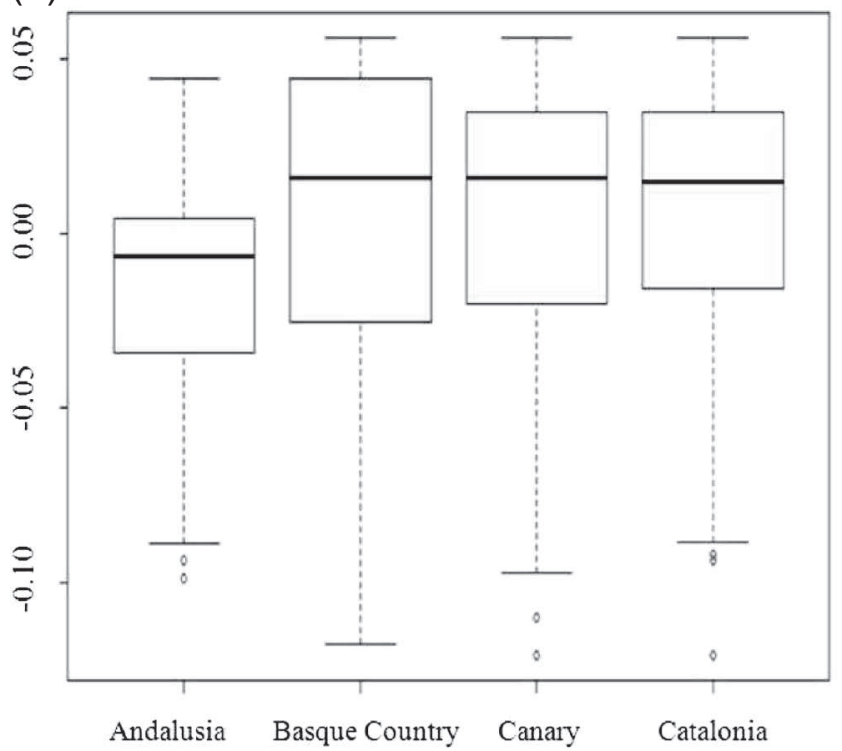

(b)

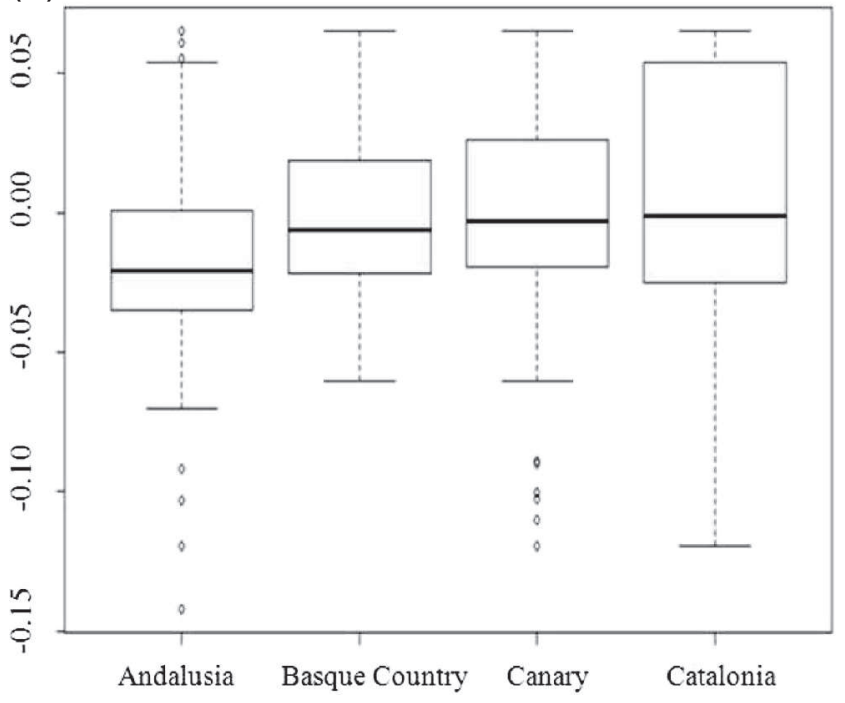

Figure 1. Box plot of component 1 among the different populations after PCA for (a) SRD5A1 and (b) SRD5A2.

ignoring possible associations with other genes and polymorphisms. Thus, the prostate cancer risk and aggressiveness conferred by certain SNPs located in 3 beta-hydroxysteroid dehydrogenase type II (HSD3B2) seem to be modified by SNPs located in SRD5A2 (Neslund-Dudas et al. 2007). In the same way, an association between SNPs in CYP17A1 (a gene encoding a key enzyme in the synthesis of androgens) and SRD5A2 has been suggested (Onen et al. 2007). Third, the number of subjects from different population varies widely. However, the fact that the main differences were not found in the population with the smallest number of patients (Basque Country, with $51 \mathrm{PCa}$ ) suggests that this limitation may not be decisive in the interpretation of results.

On the other hand, some advantages should be highlighted: (i) it includes a number of subjects sufficient to have reliable data on the distribution of these 22 SNPs in the PCa populations studied (especially for Canary and Catalonia); (ii) all subjects were male (which is the most interesting population since the $5 \alpha$-RI are contraindicated in women), then avoiding the possible bias generated by the gender; and (iii) all the determinations (13,222 in total) were performed with the same methodology, with the same batch of chips and by the same investigator, thus minimizing biases from technical origin. All the analyses were automated and there was no manual assignment of genotypes in any case.

\section{Conclusions}

Differences in distribution of genotypes in SRD5A1 and SRD5A2 within different populations of the same ethnicity could be an important factor responsible for the failure of treatments based on $5 \alpha$-RI. Our results suggest that genotypic endowment should be taken into account (especially in clinical trials including people from different ethnics) in assessing the effectiveness of these drugs.

\section{Acknowledgements}

This work was subsidized by the grant from the Instituto de Salud Carlos III (Ministerio de Economía y Competitividad from Spain), ID: PI12/01867. Almudena Valenciano has a grant from the Instituto Canario de Investigación del Cáncer (ICIC). The founders had no role in study design, data collection and analysis, decision to publish, or preparation of the manuscript. We thank the technical support from the Immunology Department (Hospital Universitario de Gran Canaria, Dr Negrín) staff: Nereida González-Quevedo and Yanira Florido-Ortega.

\section{References}

Ambrosio B., Hernandez C., Novelletto A., Dugoujon J. M., Rodriguez J. N., Cuesta P. et al. 2010 Searching the peopling of the Iberian Peninsula from the perspective of two andalusian subpopulations: a study based on Y-chromosome haplogroups $\mathrm{J}$ and E. Coll. Antropol. 34, 1215-1228.

Andriole G. L. and Kirby R. 2003 Safety and tolerability of the dual 5alpha-reductase inhibitor dutasteride in the treatment of benign prostatic hyperplasia. Eur. Urol. 44, 82-88.

Andriole G. L., Bostwick D. G., Brawley O. W., Gomella L. G., Marberger M., Montorsi F. et al. 2010 Effect of dutasteride on the risk of prostate cancer. N. Engl. J. Med. 362, 1192-1202.

Barrett J. C., Fry B., Maller J. and Daly M. J. 2005 Haploview: analysis and visualization of LD and haplotype maps. Bioinformatics 21, 263-265.

Clayton D. 2012 snpStats: SnpMatrix and XSnpMatrix classes and methods. R package version 1.8.1. Available at: http://www. bioconductor.org/packages/release/bioc/html/snpStats.html.

Cussenot O., Azzouzi A. R., Nicolaiew N., Mangin P., Cormier L., Fournier G. et al. 2007 Low-activity V89L variant in SRD5A2 is associated with aggressive prostate cancer risk: an explanation for the adverse effects observed in chemoprevention trials using 5-alpha-reductase inhibitors. Eur. Urol. 52, 1082-1087.

Edwards J. E. and Moore R. A. 2002 Finasteride in the treatment of clinical benign prostatic hyperplasia: a systematic review of randomised trials. BMC Urol. 2, 14. 
Giwercman Y. L., Abrahamsson P. A., Giwercman A., Gadaleanu V. and Ahlgren G. 2005 The 5alpha-reductase type II A49T and V89L high-activity allelic variants are more common in men with prostate cancer compared with the general population. Eur. Urol. 48, 679-685.

Henriquez-Hernandez L. A., Valenciano A., Foro-Arnalot P., Alvarez-Cubero M. J., Cozar J. M., Suarez-Novo J. F. et al. 2013a Polymorphisms in DNA-repair genes in a cohort of prostate cancer patients from different areas in Spain: heterogeneity between populations as a confounding factor in association studies. PLoS One 8, e69735.

Henriquez-Hernandez L. A., Valenciano A., Herrera-Ramos E., Lloret M., Riveros-Perez A. and Lara P. C. 2013b Highthroughput genotyping system as a robust and useful tool in oncology: experience from a single institution. Biologicals 41, 424-429.

Huggins C. 1967 Endocrine-induced regression of cancers. Cancer Res. 27, 1925-1930.

Lindstrom S., Wiklund F., Adami H. O., Balter K. A., Adolfsson J. and Gronberg H. 2006 Germ-line genetic variation in the key androgen-regulating genes androgen receptor, cytochrome P450, and steroid-5-alpha-reductase type 2 is important for prostate cancer development. Cancer Res. 66, 1107711083.

Ma Q. and Lu A. Y. 2011 Pharmacogenetics, pharmacogenomics, and individualized medicine. Pharmacol. Rev. 63, 437459.

McConnell J. D. 1995 Prostatic growth: new insights into hormonal regulation. Br. J. Urol. 76 suppl 1, 5-10.

Morcillo-Suarez C., Alegre J., Sangros R., Gazave E, de Cid R., Milne R. et al. 2008 SNP analysis to results (SNPator): a web-based environment oriented to statistical genomic analyses upon SNP data. Bioinformatics 24, 1643-1644.
Neslund-Dudas C., Bock C. H., Monaghan K., Nock N. L., Yang J. J., Rundle A. et al. 2007 SRD5A2 and HSD3B2 polymorphisms are associated with prostate cancer risk and aggressiveness. Prostate 67, 1654-1663.

Onen I. H., Ekmekci A., Eroglu M., Polat F. and Biri H. 2007 The association of 5alpha-reductase II (SRD5A2) and 17 hydroxylase (CYP17) gene polymorphisms with prostate cancer patients in the Turkish population. DNA Cell Biol. 26, 100-107.

Rando J. C., Cabrera V. M., Larruga J. M., Hernandez M., Gonzalez A. M., Pinto F. et al. 1999 Phylogeographic patterns of mtDNA reflecting the colonization of the Canary Islands. Ann. Hum. Genet. 63, 413-428.

Stanbrough M., Bubley G. J., Ross K., Golub T. R., Rubin M. A., Penning T. M. et al. 2006 Increased expression of genes converting adrenal androgens to testosterone in androgenindependent prostate cancer. Cancer Res. 66, 2815-2825.

Thomas L. N., Lazier C. B., Gupta R., Norman R. W., Troyer D. A., O'Brien S. P. et al. 2005 Differential alterations in 5alphareductase type 1 and type 2 levels during development and progression of prostate cancer. Prostate 63, 231-239.

Thompson I. M., Goodman P. J., Tangen C. M., Lucia M. S., Miller G. J., Ford L. G. et al. 2003 The influence of finasteride on the development of prostate cancer. N. Engl. J. Med. 349, 215-224.

Titus M. A., Gregory C. W., Ford 3rd O. H., Schell M. J., Maygarden S. J. and Mohler J. L. 2005 Steroid 5alpha-reductase isozymes I and II in recurrent prostate cancer. Clin. Cancer Res. 11, 4365-4371.

Walsh P. C. 2010 Chemoprevention of prostate cancer. N. Engl. J. Med. 362, 1237-1238.

Yasuda S. U., Zhang L. and Huang S. M. 2008 The role of ethnicity in variability in response to drugs: focus on clinical pharmacology studies. Clin. Pharmacol. Ther. 84, 417-423. 